\title{
3D-printed, patient-specific DIEP flap templates for preoperative planning in breast reconstruction: a prospective case series
}

\author{
Michael P. Chae ${ }^{1,2}$, David J. Hunter-Smith ${ }^{1,2}$, Ru Dee Chung ${ }^{1,2}$, Julian A. Smith ${ }^{1,2}$, Warren Matthew Rozen ${ }^{1,2 \wedge}$ \\ ${ }^{1}$ Department of Surgery, School of Clinical Sciences at Monash Health, Monash University, Level 5, E Block, Monash Medical Centre, Clayton, \\ Victoria, Australia; ${ }^{2}$ Monash University Plastic and Reconstructive Surgery Group (Peninsula Clinical School), Peninsula Health, Frankston, \\ Victoria, Australia \\ Contributions: (I) Conception and design: All authors; (II) Administrative support: DJ Hunter-Smith, JA Smith; (III) Provision of study materials or \\ patients: DJ Hunter-Smith, WM Rozen; (IV) Collection and assembly of data: All authors; (V) Data analysis and interpretation: All authors; (VI) \\ Manuscript writing: All authors; (VII) Final approval of manuscript: All authors. \\ Correspondence to: Warren Matthew Rozen. Department of Plastic and Reconstructive Surgery, Frankston Hospital, Peninsula Health, 2 Hastings \\ Road, Frankston Victoria 3199, Australia. Email: warrenrozen@hotmail.com.
}

Background Modern imaging technologies, such as computed tomographic angiography (CTA), can be
useful for preoperative assessment in deep inferior epigastric artery perforator (DIEP) flap surgery. Planning
perforator flap design can lead to improved surgical efficiency. However, current imaging modalities are
limited by being displayed on a two-dimensional (2D) surface. In contrast, a 3D-printed model provides
tactile feedback that facilitates superior understanding. Hence, we have 3D-printed patient-specific
deep inferior epigastric artery perforator (DIEP) templates, in an affordable and convenient manner, for
preoperative planning. Methods: Twenty consecutive patients undergoing 25 immediate or delayed post-mastectomy autologous breast reconstruction with DIEP or muscle-sparing transverse rectus abdominis (MS-TRAM) flaps are recruited prospectively. Using free, open-source softwares (3D Slicer, Autodesk MeshMixer, and Cura) and desktop 3D printers (Ultimaker 3E and Moment), we created a template based on a patient's abdominal wall anatomy from CTA, with holes and lines indicating the position of perforators, their intramuscular course and the DIEA pedicle.

Results: The mean age of patients was 52 [38-67]. There were 15 immediate and 10 delayed reconstructions. 3D printing time took mean 18 hours and $123.7 \mathrm{~g}$ of plastic filament, which calculates to a mean material cost of AUD 8.25. DIEP templates accurately identified the perforators and reduced intraoperative perforator identification by 7.29 minutes $(\mathrm{P}=0.02)$. However, the intramuscular dissection time was not affected $(\mathrm{P}=0.34)$. Surgeons found the template useful for preoperative marking $(8.6 / 10)$ and planning $(7.9 / 10)$, but not for intramuscular dissection (5.9/10). There were no immediate flap-related complications.

Conclusions: Our 3D-printed, patient-specific DIEP template is accurate, significantly reduces intraoperative perforator identification time and, hence, may be a useful tool for preoperative planning in autologous breast reconstruction.

Keywords: 3D printing; surgical template; deep inferior epigastric artery perforator (DIEP); autologous breast reconstruction; preoperative planning

Submitted Apr 20, 2021. Accepted for publication May 26, 2021.

doi: $10.21037 /$ gs-21-263

View this article at: https://dx.doi.org/10.21037/gs-21-263

^ ORCID: 0000-0002-4092-182X. 


\section{Introduction}

The incidence of breast cancer diagnosis has been rising steadily (1) and an increasing number of women are opting for post-mastectomy reconstruction (2). In comparison to implant-based techniques, autologous breast reconstruction based on deep inferior epigastric artery perforator (DIEP) flap yields long-lasting, natural-appearing outcome and is, hence, considered the gold standard reconstruction (3-9).

Due to high individual variation in the vascular anatomy of the DIEA perforators (10), computed tomographic angiography (CTA) is performed to help select ideal perforator and flap design preoperatively (11-16). Advances in the hardware of CTA, such as an increasing number of detector rows (17), and software, such as OsiriX (Pixmeo, Geneva, Switzerland) (18), have ensured its precision, fast speed and reliability.

As a result, the use of CTA in DIEP flap planning has led to reduced postoperative flap complications, such as fat necrosis, flap loss and donor site morbidity $(12,19-22)$. Interestingly, Smit et al. have reported a significant reduction in operating time using CTA in DIEP reconstructions (264 vs. 354 mins, $\mathrm{P}<0.001)$ (11). However, Rozen $e t$ al. have reported no statistically significant reduction in the total operating time of both unilateral and bilateral cases ( $\mathrm{P}=0.57$ and 0.079 , respectively) (12). This may be attributed to the fact that current imaging modalities are limited by being displayed on a two-dimensional (2D) surface like a computer screen. Novel ways of utilising the CTA data, such as 3D printing, may enable intuitive spatio-temporal understanding of the involved anatomical structures.

In the last decade, 3D printing has become affordable and readily accessible $(23,24)$. In plastic and reconstructive surgery, $3 \mathrm{D}$ printing appears most useful in preoperative planning, designing intraoperative guidance devices, patient education, and building customized implants (25-27). Recently, we have developed an affordable and convenient method of 3D printing for clinicians using free, opensource softwares and desktop 3D printers (28-31). Using this technique, we have 3D-printed patient-specific DIEP templates and utilized them in 20 consecutive patients receiving autologous breast reconstruction with abdominalwall based free flaps.

This prospective study aims to assess the feasibility and usefulness of 3D designed DIEP perforator templates as a practical tool for DIEP breast reconstruction.

\section{Methods}

In this prospective case series of 25 abdominal wall-based flaps in 20 patients over 12-month period (June 2016 to June 2017), we use routine CTA scan data to create patientspecific DIEP templates for preoperative planning. The study conformed to the provisions of the Declaration of Helsinki (as revised in 2013). Ethical approval was obtained through Peninsula Health HREC, approval number $\mathrm{PH} / 3 \mathrm{DP}$, and all participants gave informed consent before taking part.

\section{$C T A$}

CTA was performed using standardized "single-volume" acquisition technique that ensures maximal image quality and minimal radiation exposure, as previously described by Rozen et al. (32). Siemens SOMATOM Sensation 64 multidetector row computed tomography scanner (Siemens Medical Solutions, Erlangen, Germany) was used and the scan parameters are summarized in Table 1.

\section{Design of the DIEP template}

CTA data is exported from the scanner in DICOM (Digital Imaging and COmmunications in Medicine) format and is processed using free, open-source software (see Figure 1): 3D Slicer (Surgical Planning Laboratory, Boston, MA, USA), Autodesk MeshMixer (Autodesk, Inc., San Rafael, CA, USA), and Cura (Ultimaker, Geldermalsen, Netherlands).

Following this, a 3D image of the patient's abdominal wall with perforations indicating the location of perforators is created from the DICOM files in 3D Slicer. Using the "threshold" function, a range of Hounsfield unit-derived arbitrary values can be selected to automatically generate a $3 \mathrm{D}$ image of the abdominal wall. Scrolling through the axial slices, using "RectangleEffect" tool and erase function, holes and lines are created in the 3D image at the location of DIEA perforators, their intramuscular course and the DIEA pedicle. Similarly, a notch in the 3D image is created at pubic symphysis. The final 3D image is exported in STL (standard tessellation language) format.

In Autodesk MeshMixer, the STL file is made suitable for clinical application. Using "SphereDisc" brush tool, the perforators, their intramuscular course and the DIEA are enlarged to enable a marking pen to fit. Then, the 3D image 
Table 1 Computed tomographic angiography scan parameters

\begin{tabular}{ll}
\hline Parameters & Characteristics \\
\hline Scanner & Siemens SOMATOM Sensation 64 \\
Scan type & Helical multi-detector row CT angiography \\
Slice thickness & 64 detector row $\times 0.6 \mathrm{~mm}$ collimator width \\
Helical detector pitch & 0.9 \\
Gantry rotation speed & $0.37 \mathrm{sec}$ \\
Tube potential & $120 \mathrm{kV}$ \\
Tube current & $180 \mathrm{~mA}$ \\
Contrast & Omnipaque $350100 \mathrm{~mL}$ IV injection 4 ml per second \\
Scanning range & Pubic symphysis to $4 \mathrm{~cm}$ above umbilicus \\
Scanning direction & Caudo-cranial \\
Bolus tracking & +100 HU from common femoral artery with minimal delay \\
Automatic dose modulation (Siemens Care Dose 4D) & Disabled \\
Imaging reconstruction & $1 \mathrm{~mm} / 0.75 \mathrm{~mm}$ overlapping axial images \\
\hline
\end{tabular}
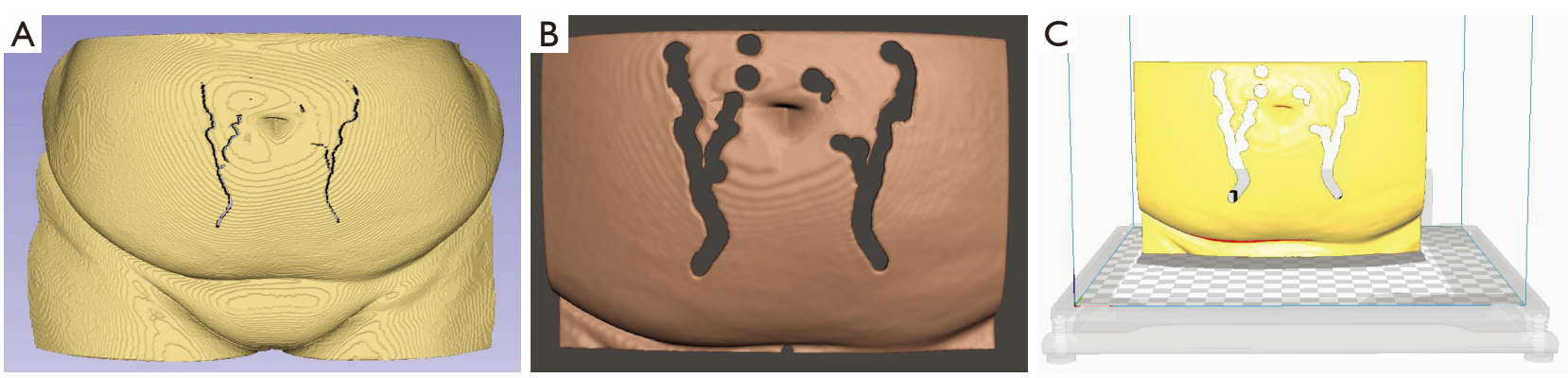

Figure $13 \mathrm{D}$ printing software. (A) Using 3D Slicer software (Surgical Planning Laboratory, Boston, MA USA), CTA scan data is converted into a 3D image of the abdominal wall and holes and lines are placed appropriately to indicate the location of DIEA perforators, their intramuscular course and the DIEA pedicle. (B) Using Autodesk MeshMixer software (Autodesk, Inc., San Rafael, CA USA), the 3D image is cropped into appropriate size and the holes/lines are enlarged to fit marking pens. (C) Using Cura software (Ultimaker, Geldermalsen, Netherlands), the final file is converted to a 3D printer-friendly file. CTA, computed tomographic angiography; DIEA, deep inferior epigastric artery.

is cropped around four edges to fit inside a $3 \mathrm{D}$ printer. Using "separate" function, anterior surface of the 3D image is isolated and then, using "extrude" function, it is thickened $5 \mathrm{~mm}$ into a physical template. Finally, using "smooth" function, the file is smoothed out and then exported in STL format.

In Cura, this final STL file is transformed into a 3D printer-friendly file. 3D slicing software, like Cura, automatically generates support structures and creates an instruction for a $3 \mathrm{D}$ printer to follow. This file is exported in G-code format via an external storage device.

\section{$3 D$ printing}

The templates are 3D-printed in thermoplastic polylactic acid (PLA) filament using one of our two desktop fused filament fabrication (FFF) 3D printers: Ultimaker 3 Extended 3D printer (Ultimaker, Geldermalsen, Netherlands) and Moment 3D printer (Moment, Seoul, South Korea). They cost approximately AUD 6,000 and 

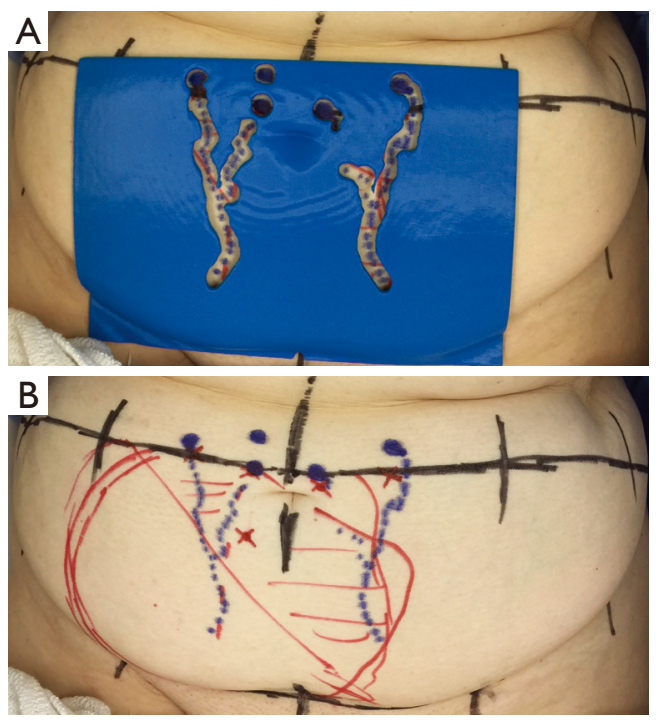

Figure 2 Clinical application of the 3D-printed DIEP template in use. The template was orientated on the abdomen by the umbilicus, notch at the pubic symphysis and the abdominal skin crease (A). Markings from the template were used for flap design and the skin island (B). DIEP, deep inferior epigastric artery perforator.

3,000 , respectively. The latter is used to print smaller-sized templates.

\section{Patient recruitment}

All patients undergoing postmastectomy autologous breast reconstruction with abdominal wall-based free flaps, regardless of whether immediate or delayed, unilateral or bilateral, and DIEP or MS-TRAM flaps, were recruited from three university-affiliated hospital networks. Exclusion criteria include contraindication to intravenous contrast preventing preoperative investigation with CTA, patient decline and pregnancy. Historical control cases were derived from reconstructions performed at the same institutions over the preceding 12 months (June 2015 - June 2016).

\section{Accuracy of the DIEP template}

In order to assess the templates accuracy and reliability, perforator distance from the base of umbilicus (horizontal \& vertical) was measured using a calibrated calliper. We selected the biggest perforator from each hemi-abdomen. This step was repeated using perforators detected using handheld Doppler probes (33) and reported measurements from the patients CTA (34). Intraoperative measurement of the perforator distances was made using a sterile metal ruler and performed just prior to the flap being disconnected at the DIEA origin. This was achieved with measurements from CTA, with Doppler and with the template applied topically to the abdominal wall directly (see Figure 2).

\section{Utility of the DIEP template: operating time}

In order to assess the potential role of the template in improving intraoperative perforator identification, we measured the time taken during suprafascial flap dissection. Recently, Marsh et al. from Chelmsford, UK, described a technique to divide the DIEP flap reconstruction procedure into 101 individual steps (35). As a result, we have measured the time taken during the steps 24 ("lateral raise of flap off rectus fascia with handheld diathermy to just lateral to lateral row perforator") and 25 ["dissection down to and identification of perforator (match to CT) using bipolar diathermy and/or McIndoe's dissection scissors"].

Similarly, in order to assess its potential role in improving intramuscular dissection, we have measured the time taken during steps 29 ["subfascial/intramuscular dissection (muscle relaxant or lignocaine) using McIndoe dissecting forceps and bipolar"] and 30 ("submuscular dissection of perforator") (35).

Since June 2015 , at our institution, we have been routinely recording the operating time of the Chelmsford 101 individual steps in free DIEP flap breast reconstructions. As a result, our outcomes from the current study could be accurately correlated to the historical control.

\section{Utility of the DIEP template: surgeon perception surveys}

After each operation, both primary and second surgeons and surgical trainees have completed a 5-part survey that assesses their perceived utility of the template on a 10 -score visual analogue scale. It consisted of the following questions: "how useful was the device in preoperative marking?", "how useful was the device in preoperative planning?", "how useful was the device in intramuscular dissection?", "did it change your management?" and "would you use it again?".

\section{Statistical analysis}

Perforator distances from the template, handheld Doppler probes, CTA report, and intraoperative findings are 
Table 2 Summary of responses from the surgeon perception survey and comparison of responses between flap-raising surgeons against the second surgeons and surgeons against surgical trainees

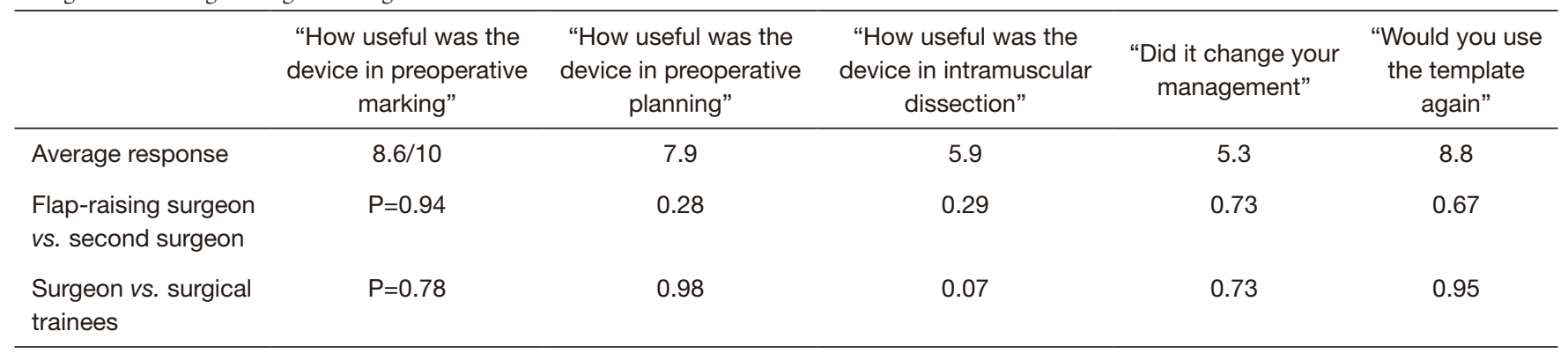

recorded using calibrated callipers and sterile rulers. They are rounded to the nearest $1 \mathrm{~mm}$. Intraoperative perforator identification time and intramuscular dissection time are recorded using a stopwatch and rounded to the nearest 1 second. The comparative analysis was performed using Stata statistical software package (StataCorp, College Station, TX, USA). The perforator distances were analysed using Kruskal-Wallis equality-of-populations rank test while the surgical times and the survey responses were analysed using the Student's $t$-test. A P value of less than 0.05 was accepted as statistically significant.

\section{Results}

A total of 20 patient-specific DIEP templates were $3 \mathrm{D}$-printed for 25 consecutive autologous breast reconstructions in 20 patients between July 2016 - June 2017 (12 months). Mean age of the patients was 52 (range: 38-67) and the mean BMI was 27.8 (21-36.4). Immediate reconstruction made up 15 out of 25 flaps (60\%), unilateral reconstruction 15 out of 20 cases (75\%), and DIEP flaps 18 out of 25 flaps (72\%). Historical control consisted of 9 consecutive autologous reconstructions in 7 patients.

\section{$3 D$ printing}

Each template took mean 18.03 hours to 3D print and used $123.7 \mathrm{~g}$ of filament. Given that $750 \mathrm{~g}$ of PLA filament costs approximately US\$39, mean material cost was less than US\$6.50 per template.

\section{Accuracy of the DIEP template}

The template accurately identified DIEA perforators and is as accurate as current gold standard imaging modalities: handheld Doppler and CTA. There was no statistical difference between the template and intraoperative measurements (horizontal and vertical distances; $\mathrm{P}=0.09$ and 0.87 , respectively). Similarly, there was no statistical difference between the template, handheld Doppler and CTA (horizontal and vertical distances; $\mathrm{P}=0.42$ and 0.74 , respectively).

\section{Utility of the DIEP template: operating time}

The template had a dissimilar impact on different stages of the operation. Mean intraoperative perforator identification time was significantly reduced by 7.29 minutes (15.07 vs. 22.36 mins; $\mathrm{P}=0.02)$. However, there was no statistically significant difference in mean intramuscular dissection time (93.95 vs. 79.62 mins; $\mathrm{P}=0.34$ ).

\section{Utility of the DIEP template: Surgeon perception survey}

The template was useful for preoperative marking (mean score: 8.6/10) and planning (7.9/10) (Table 2). However, it was not useful for intramuscular dissection (5.9/10) and, as a result, did not influence the clinical management significantly (5.3/10). Encouragingly, the surgeons appeared enthused about its potential and were keen to use the template again (8.8/10). When the responses were compared between flapraising surgeons and the second surgeons, there was no difference. Similarly, there was no statistical differences between surgeons and surgical trainees. Interestingly, there was a trend in the difference between surgeons and surgical trainees in their perceive utility of the template in intramuscular dissection ( 4.7 vs. $6.3, \mathrm{P}=0.07)$. However, since the response from trainees was equally low (6.3/10), it is most likely that trainees had similar doubts about its usefulness in intramuscular dissection. 


\section{Clinical outcomes}

There was no immediate flap-related postoperative complications. Mean length of stay was 6.4 days (range: 5-8). At 3-month follow-up, there was no reported donorsite morbidity clinically, such as abdominal wall bulge and ventral hernia.

\section{Discussion}

In the current prospective case series, we demonstrate our technique of creating a 3D-printed, patient-specific DIEP template, assess its accuracy and utility and illustrate outcomes to our historical control.

Usually, a radiologist would report from CTA the diameter of suitable perforators and their location in horizontal and vertical distances from the umbilicus. This is marked out on the patient by the surgeon preoperatively and confirmed using a handheld Doppler probe. Unsurprisingly, during observation, transcription and interpretation of the report, errors can be introduced and compromise efficiency (36). Thus, Miranda et al. have proposed, in their proof-of-concept study, a method of creating patient-specific 2D DIEP templates using transparent acetate sheets, punch biopsy holes, and coronal images from the CTA (36). However, this technique is rather cumbersome and is significantly susceptible to observation and transcription errors. Moreover, the flat acetate sheet ignores the curved contour of the abdominal wall. To this effect, $3 \mathrm{D}$ printing can be useful.

Aided by expiration of key patents, 3D printing, also known as additive manufacturing or rapid prototyping, has become affordable and readily accessible for clinicians in the last decade $(23,25-27,37)$. In comparison to the traditional manufacturing process, $3 \mathrm{D}$ printing enables easy customization in a cost-efficient and convenience manner. In plastic and reconstructive surgery, 3D printing appears most useful in preoperative planning, designing intraoperative guidance devices, patient education, and building customized implants (25-27). Using free, opensource softwares (i.e., 3D Slicer, Autodesk MeshMixer, and Cura) and desktop 3D printers (i.e., Ultimaker 3E and Moment), we have produced each template at a material cost of less than AUD 10.

The template accurately identifies the position of DIEA perforators, their intramuscular course and the DIEA pedicle. Furthermore, it improves preoperative marking and planning, leading to statistically quicker intraoperative identification of the perforators. This may be because the template removes the guesswork and interpretation errors from traditional written CTA reports and is more intuitive to apply since they lie accurately on the abdomen. Furthermore, the tactile feedback from templates likely enhances visuospatial understanding of the involved perforator anatomy, leading to more confident dissection (38). However, despite being statistically significant, the reduction of 7.29 minutes may be too small to be clinical significant. Notably, the template appears not useful for intramuscular dissection, which is arguably one of the most technically challenging aspects of DIEP flaps. This is most likely because despite being $3 \mathrm{D}$-printed, clinical information is essentially embossed in $2 \mathrm{D}$ on to the template.

$3 \mathrm{D}$ printing the entire course of a DIEA perforator and its surrounding soft tissues, similar to what Mehta et al. have reported in a proof-of-concept study, may be more useful for intramuscular dissection (39). However, their technique would have been difficult to reproduce for routine clinical application due to software- and hardwarerelated issues. Using any latest imaging software, it remains difficult to differentiate DIEA perforators from the rectus abdominis in CTA both visually and digitally, especially in patients with physiologically smaller vessels, without significantly increasing the contrast dose and radiation. Using MRA, the image resolution is even poorer $(5.0 \mathrm{vs}$. $0.5 \mathrm{~mm}$ slice thickness) since scans have to be performed quickly to prevent motion artefacts. Recent advances in MRA technology can account for motion artefacts without compromising image quality, but they are not yet available widely (40). In contrast to the multi-colour, multi-material, industrial-grade 3D printer used by Mehta et al. that costs in excess of AUD 100,000, our desktop 3D printers (Ultimaker $3 \mathrm{E}$, Moment) can only print in single material, making it difficult to print intramuscular course of DIEA perforators. As a result, most clinicians currently outsource $3 \mathrm{D}$ printing at a cost of more than AUD 1,000 per model.

\section{Conclusions}

We demonstrate that our 3D-printed, patient-specific DIEP template accurately identifies DIEA perforators, and significantly reduces intraoperative perforator identification time by, albeit 7.29 minutes, and, as a result, it may become a useful tool in preoperative marking and planning.

\section{Acknowledgments}

The current work has been accepted and presented at 
the $9^{\text {th }}$ Congress of World Society for Reconstructive Microsurgery in Seoul, South Korea, and at the Australasian Society of Plastic Surgery's Plastic Surgery Congress 2017, Gold Coast, Australia.

Funding: None.

\section{Footnote}

Data Sharing Statement: Available at https://dx.doi. org/10.21037/gs-21-263

Conflicts of Interest: All authors have completed the ICMJE uniform disclosure form (available at https://dx.doi. org/10.21037/gs-21-263). Warren M. Rozen serves as an unpaid Associate Editor of Gland Surgery from Mar 2018 to Feb 2023. The other authors have no conflicts of interest to declare.

Ethical Statement: The authors are accountable for all aspects of the work in ensuring that questions related to the accuracy or integrity of any part of the work are appropriately investigated and resolved. The study conformed to the provisions of the Declaration of Helsinki (as revised in 2013). Ethical approval was obtained through Peninsula Health HREC, approval number PH/3DP. The participants gave informed consent before taking part.

Open Access Statement: This is an Open Access article distributed in accordance with the Creative Commons Attribution-NonCommercial-NoDerivs 4.0 International License (CC BY-NC-ND 4.0), which permits the noncommercial replication and distribution of the article with the strict proviso that no changes or edits are made and the original work is properly cited (including links to both the formal publication through the relevant DOI and the license). See: https://creativecommons.org/licenses/by-nc-nd/4.0/.

\section{References}

1. DeSantis C, Ma J, Bryan L, et al. Breast cancer statistics, 2013. CA Cancer J Clin 2014;64:52-62.

2. Wong SM, Freedman RA, Sagara Y, et al. Growing Use of Contralateral Prophylactic Mastectomy Despite no Improvement in Long-term Survival for Invasive Breast Cancer. Ann Surg 2017;265:581-9.

3. Jeevan R, Browne JP, Gulliver-Clarke C, et al. Surgical Determinants of Patient-Reported Outcomes following Postmastectomy Reconstruction in Women with Breast
Cancer. Plast Reconstr Surg 2017;139:1036e-45e.

4. Patel NG, Ramakrishnan V. Microsurgical Tissue Transfer in Breast Reconstruction. Clin Plast Surg 2017;44:345-59.

5. Matthews H, Carroll N, Renshaw D, et al. Predictors of satisfaction and quality of life following post-mastectomy breast reconstruction. Psychooncology 2017;26:1860-5.

6. Spear SL, Onyewu C. Staged breast reconstruction with saline-filled implants in the irradiated breast: recent trends and therapeutic implications. Plast Reconstr Surg 2000;105:930-42.

7. Blondeel PN. One hundred free DIEP flap breast reconstructions: a personal experience. Br J Plast Surg 1999;52:104-11.

8. Kroll SS. Why autologous tissue? Clin Plast Surg 1998;25:135-43.

9. Allen RJ, Treece P. Deep inferior epigastric perforator flap for breast reconstruction. Ann Plast Surg 1994;32:32-8.

10. Ireton JE, Lakhiani C, Saint-Cyr M. Vascular anatomy of the deep inferior epigastric artery perforator flap: a systematic review. Plast Reconstr Surg 2014;134:810e-21e.

11. Smit JM, Dimopoulou A, Liss AG, et al. Preoperative $\mathrm{CT}$ angiography reduces surgery time in perforator flap reconstruction. J Plast Reconstr Aesthet Surg 2009;62:1112-7.

12. Rozen WM, Anavekar NS, Ashton MW, et al. Does the preoperative imaging of perforators with CT angiography improve operative outcomes in breast reconstruction? Microsurgery 2008;28:516-23.

13. Clavero JA, Masia J, Larranaga J, et al. MDCT in the preoperative planning of abdominal perforator surgery for postmastectomy breast reconstruction. AJR Am J Roentgenol 2008;191:670-6.

14. Masia J, Larranaga J, Clavero JA, et al. The value of the multidetector row computed tomography for the preoperative planning of deep inferior epigastric artery perforator flap: our experience in 162 cases. Ann Plast Surg 2008;60:29-36.

15. Rozen WM, Ashton MW, Grinsell D, et al. Establishing the case for CT angiography in the preoperative imaging of abdominal wall perforators. Microsurgery 2008;28:306-13.

16. Rosson GD, Williams CG, Fishman EK, et al. 3D CT angiography of abdominal wall vascular perforators to plan DIEAP flaps. Microsurgery 2007;27:641-6.

17. Pratt GF, Rozen WM, Chubb D, et al. Preoperative imaging for perforator flaps in reconstructive surgery: a systematic review of the evidence for current techniques. Ann Plast Surg 2012;69:3-9. 
18. Chae MP, Hunter-Smith DJ, Rozen WM. Comparative study of software techniques for 3D mapping of perforators in deep inferior epigastric artery perforator flap planning. Gland Surg 2016;5:99-106.

19. Tong WM, Dixon R, Ekis H, et al. The impact of preoperative CT angiography on breast reconstruction with abdominal perforator flaps. Ann Plast Surg 2012;68:525-30.

20. Masia J, Kosutic D, Clavero JA, et al. Preoperative computed tomographic angiogram for deep inferior epigastric artery perforator flap breast reconstruction. J Reconstr Microsurg 2010;26:21-8.

21. Scott JR, Liu D, Said H, et al. Computed tomographic angiography in planning abdomen-based microsurgical breast reconstruction: a comparison with color duplex ultrasound. Plast Reconstr Surg 2010;125:446-53.

22. Casey WJ, 3rd, Chew RT, Rebecca AM, et al. Advantages of preoperative computed tomography in deep inferior epigastric artery perforator flap breast reconstruction. Plast Reconstr Surg 2009;123:1148-55.

23. Sachs EM, Haggerty JS, Cima MJ, et al. Threedimensional printing techniques. US Patent No. 5,204,055:April 20, 1993.

24. Hull CW. Apparatus for production of threedimensional objects by stereolithography. US Patent No. 4,575,330:March 11, 1986.

25. Kamali P, Dean D, Skoracki R, et al. The Current Role of Three-Dimensional (3D) Printing in Plastic Surgery. Plast Reconstr Surg 2016;137:1045-55.

26. Chae MP, Rozen WM, McMenamin PG, et al. Emerging Applications of Bedside 3D Printing in Plastic Surgery. Front Surg 2015;2:25.

27. Gerstle TL, Ibrahim AM, Kim PS, et al. A plastic surgery application in evolution: three-dimensional printing. Plast Reconstr Surg 2014;133:446-51.

28. Chae MP, Hunter-Smith DJ, De-Silva I, et al. FourDimensional (4D) Printing: A New Evolution in Computed Tomography-Guided Stereolithographic Modeling. Principles and Application. J Reconstr Microsurg 2015;31:458-63.

29. Cabalag MS, Chae MP, Miller GS, et al. Use of three-

Cite this article as: Chae MP, Hunter-Smith DJ, Chung RD, Smith JA, Rozen WM. 3D-printed, patient-specific DIEP flap templates for preoperative planning in breast reconstruction: a prospective case series. Gland Surg 2021;10(7):2192-2199. doi: $10.21037 /$ gs-21-263 dimensional printed 'haptic' models for preoperative planning in an Australian plastic surgery unit. ANZ J Surg 2017;87:1057-9.

30. Chae MP, Lin F, Spychal RT, et al. 3D-printed haptic "reverse" models for preoperative planning in soft tissue reconstruction: a case report. Microsurgery 2015;35:148-53.

31. Chae MP, Hunter-Smith DJ, Spychal RT, et al. 3D volumetric analysis for planning breast reconstructive surgery. Breast Cancer Res Treat 2014;146:457-60.

32. Rozen WM, Whitaker IS, Stella DL, et al. The radiation exposure of Computed Tomographic Angiography (CTA) in DIEP flap planning: low dose but high impact. J Plast Reconstr Aesthet Surg 2009;62:e654-5.

33. Taylor GI, Doyle M, McCarten G. The Doppler probe for planning flaps: anatomical study and clinical applications. Br J Plast Surg 1990;43:1-16.

34. Rozen WM, Ashton MW, Stella DL, et al. The accuracy of computed tomographic angiography for mapping the perforators of the DIEA: a cadaveric study. Plast Reconstr Surg 2008;122:363-9.

35. Marsh D, Patel NG, Rozen WM, et al. Three routine free flaps per day in a single operating theatre: principles of a process mapping approach to improving surgical efficiency. Gland Surg 2016;5:107-14.

36. Miranda BH, Pywell M, Floyd D. A preoperative marking template for deep inferior epigastric artery perforator flap perforators in breast reconstruction. Arch Plast Surg 2014;41:171-3.

37. Hull CW. Method for production of three-dimensional objects by stereolithography. US Patent No. 4, 929,402: May 29, 1990.

38. Way TP, Barner KE. Automatic visual to tactile translation--Part II: Evaluation of the TACTile Image Creation System. IEEE Trans Rehabil Eng 1997;5:95-105.

39. Mehta S, Byrne N, Karunanithy N, et al. 3D printing provides unrivalled bespoke teaching tools for autologous free flap breast reconstruction. J Plast Reconstr Aesthet Surg 2016;69:578-80.

40. Ma C, Clifford B, Liu Y, et al. High-resolution dynamic 31 P-MRSI using a low-rank tensor model. Magn Reson Med 2017;78:419-28. 